Radial and Nonradial Pulsations as Probes of Stellar Physics

ASP Conference Series, Vol. 259, 2002

C. Aerts, T.R. Bedding, \& J. Christensen-Dalsgaard, eds.

\title{
Frequency Analysis of $\delta$ Scuti and RR Lyrae Stars in the OGLE-1 Database
}

\author{
P. Moskalik \\ Copernicus Astronomical Centre, Warsaw, Poland \\ E. Poretti \\ Osservatorio Astronomico di Brera, Merate, Italy
}

\begin{abstract}
We discuss the results of a systematic search for multiperiodic pulsations in Galactic Bulge $\delta$ Scuti and RR Lyrae stars. Six "normal" double-mode variables pulsating in two radial modes have been identified ( $5 \delta$ Scuti-type and 1 RR Lyrae-type). In $37 \mathrm{RR}$ Lyrae stars secondary periodicities very close to the primary pulsation frequency have been detected. These periodicities correspond to nonradial modes of oscillation. They are found in $\sim 23 \%$ of RRab and in $\sim 3 \%$ of RRc variables of our sample.
\end{abstract}

\section{Introduction}

The Optical Gravitational Lensing Experiment (OGLE; Udalski et al., 1992) is devoted to a search for dark matter in our galaxy using microlensing phenomena. As a by-product of this program, extensive photometry of variable stars in the Galactic Bulge has been accumulated (Udalski et al., 1994, 1995, 1996, 1997). We have performed a systematic frequency analysis of RR Lyrae and $\delta$ Scuti stars of this sample. The results for monoperiodic variables are presented elsewhere (Poretti, 2001). Here, we discuss the main properties of the identified multiperiodic pulsators.

\section{Search for multiperiodicity}

The OGLE- 1 database contains $215 \mathrm{RR}$ Lyrae and $53 \delta$ Scuti variables. The data span $\sim 900$ days, with typically 130-150 I-band measurements per star. As a first step of analysis, the lightcurve is fitted with the Fourier sum of the form

$$
\mathrm{m}_{\mathrm{I}}(t)=\mathrm{A}_{0}+\sum_{i=1}^{N} \mathrm{~A}_{i} \cos \left(\frac{2 \pi}{\mathrm{P}_{1}} t+\phi_{i}\right)
$$

with primary pulsation period $P_{1}$ being adjusted as well. Next, the search for secondary periodicities is performed. Two different methods are applied:

a) we compute Fourier power spectrum of the residuals of the fit 
b) we supplement the fitting formula with an additional cosine term with a trial period $\mathrm{P}_{2}$. We then fit the data for different values of $\mathrm{P}_{2}$, searching for the value that reduces the dispersion of the fit in a significant way.

Both methods yield the same results. As a third step, the Fourier fit with two identified frequencies and their detectable linear combinations is performed. The search for additional periodicities is then repeated. The process is stopped when no significant additional term appears.

\section{Canonical double-mode pulsators}

We have identified 6 "normal" double-mode variables, five among $\delta$ Scuti stars and one (BW7 V30) among RR Lyrae stars. They are listed in Table1. The period ratios are very typical and correspond to the ratios of the first two radial overtones. The only exception is BWC V82 with $\mathrm{P}_{2} / \mathrm{P}_{1}=0.55$, where a higher order radial mode (third overtone) has to be invoked.

Table 1. Canonical double-mode variables

\begin{tabular}{lcc}
\hline Star & $\mathrm{P}_{1}[$ day $]$ & $\mathrm{P}_{2} / \mathrm{P}_{1}$ \\
\hline BW2 V142 & 0.066 & 0.778 \\
BW9 V192 & 0.076 & 0.754 \\
BW1 V207 & 0.085 & 0.774 \\
BW1 V109 & 0.106 & 0.774 \\
BWC V82 & 0.161 & 0.550 \\
\hline BW7 V30 & 0.362 & 0.743 \\
\hline
\end{tabular}

\section{Blazhko effect: nonradial oscillations in RR Lyrae stars}

In $37 \mathrm{RR}$ Lyrae variables a different type of multiperiodicity is found - additional peaks very close to the primary pulsation frequency are present. Their amplitudes are usually below $0.06 \mathrm{mag}$. The secondary frequencies are well-resolved within our dataset and are not due to a secular period variability. Their beating with the primary (radial) pulsation results in an apparent long-term amplitude and phase modulation, a phenomenon referred to as the Blazhko effect.

This new multiperiodic behaviour comes in two different flavours: we see either a single secondary peak, forming a doublet with the primary frequency (1 RRc and 27 RRab stars) or a pair of secondary peaks, which together with the primary frequency form an equidistant triplet centered on the primary peak (1 RRc and 8 RRab stars).

While the frequency triplet can result from periodic amplitude and/or phase modulation of a purely radial pulsation, such a process cannot produce a doublet. The observed period ratios $\left(\mathrm{P}_{2} / \mathrm{P}_{1}=0.95-1.02\right)$ are not compatible with excitation of two radial modes. The unavoidable conclusion is that a secondary component of the doublet must correspond to a nonradial mode of oscillation. 


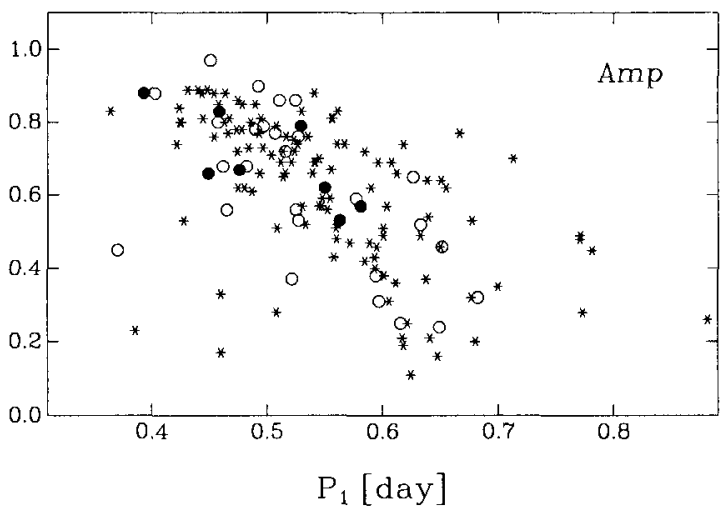

Figure 1. Amplitudes of Galactic Bulge RRab stars (as given by OGLE team). Asterisks: monoperiodic variables, open circles: variables with frequency doublets, filled circles: variables with frequency triplets.

In Table 2 we present the statistics of variables with closely spaced frequencies for the Galactic Bulge sample, compared with the numbers derived for the LMC (Alcock et al., 2000; Welch et al., these proceedings). In both populations, close doublets/triplets occur more frequently in fundamental-mode pulsators than in overtone pulsators. Interestingly, the fraction of multiperiodic RRab stars is two times higer in the Galactic Bulge than in the LMC. It is tempting to speculate that the difference in the incidence rate is related to difference in metallicity of the two populations. This hypothesis can be tested when photometry of RR Lyrae stars in the SMC is analysed.

Table 2. Incidence rate of RR Lyrae variables with closely spaced frequencies in the Galactic Bulge and in the LMC. Standard deviations calculated assuming Poisson distribution.

\begin{tabular}{lcr}
\hline Type & Galactic Bulge & \multicolumn{1}{c}{ LMC } \\
\hline fundamental-mode pulsators (RRab) & $23.2 \pm 3.9 \%$ & $10.2 \pm 0.8 \%$ \\
overtone pulsators (RRc) & $3.1 \pm 2.2 \%$ & $3.9 \pm 0.5 \%$ \\
\hline
\end{tabular}

With the sample of 35 multiperiodic RRab stars, we can discuss the group properties of this type of variables. In Fig. 1 we plot amplitudes of the Galactic Bulge RRab stars as a function of their periods. The presence of secondary frequencies has no apparent effect on the pulsation amplitude. We note that close frequency doublets are detected with roughly the same probability at all periods represented in this sample. The occurrence of triplets, on the other hand, seems to be limited to $\mathrm{P}<0.6$ day. 


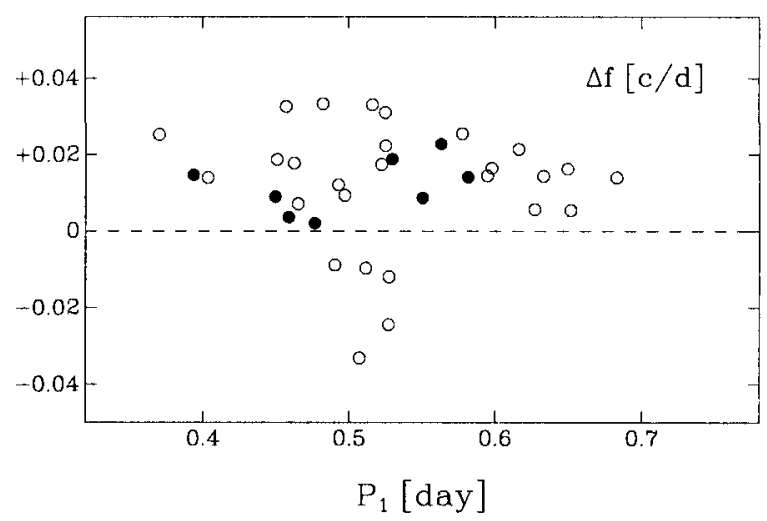

Figure 2. Frequency difference, $\Delta \mathrm{f}$, for Galactic Bulge multiperiodic RRab stars. Symbols the same as in Fig. 1.

Fig. 2 shows the frequency separation $\Delta f=f_{2}-f_{1}(f=1 / P)$ for multiperiodic $\mathrm{RRab}$ variables identified in this work. For $80 \%$ of all doublets $\Delta f$ is positive, corresponding to secondary frequency being higher than the primary one. An identical distribution of $\Delta f$ has also been found for RRab stars in the LMC (Welch et al., these proceedings). The negative values of $\Delta \mathrm{f}$ occur only in a narrow range of periods between 0.49 day and 0.53 day. Curiously, this particular period range seems to be avoided by stars with triplets.

The frequency separation in the triplets tends to be slightly smaller than in the doubles. For both triplets and doublets, the separation $\Delta \mathrm{f}$ is significantly smaller than in the overtone RR Lyrae variables of the LMC (Alcock et al., 2000).

Acknowledgments. This work has been supported by Polish KBN grants 2 P03D 00220 and 5 P03D 01220.

\section{References}

Alcock, C., Allsman, R., Alves, D. R., et al. 2000, ApJ, 542, 257

Poretti, E. 2001, A\&A, 371, 986

Udalski, A., Szymański, M., Kalużny, J. \& Kubiak, M. 1993, Acta Astron., 42, 253

Udalski, A., Kubiak, M., Szymański, M., et al. 1994, Acta Astron., 44, 317

Udalski, A., Olech, A., Szymański, M., et al. 1995, Acta Astron., 45, 433

Udalski, A., Olech, A., Szymański, M., et al. 1996, Acta Astron., 46, 51

Udalski, A., Olech, A., Szymański, M., et al. 1997, Acta Astron., 47, 1 